\title{
Development of acidophilus milk via selected probiotics \& prebiotics using artificial neural network
}

\author{
Zeynab Raftani Amiri ${ }^{1 *}$, Pratima Khandelwal' ${ }^{2}$ B. R. Aruna ${ }^{2}$ \\ ${ }^{1}$ Department of Food Science and Technology, Sari Agricultural Sciences and Natural Resources University, Sari, Iran; \\ ${ }^{2}$ P.G. Department of Biotechnology, Brindavan College, Bangalore, India. \\ Email:irajarash@rediffmail.com
}

Received 27 April 2010; revised 30 May 2010; accepted 7 June 2010.

\begin{abstract}
Commercial interest in functional foods containing probiotic strains has consistently increased due to the awareness of gut health. Recent advancements are leading to development of synbiotic foods, containing prebiotics and probiotics bearing synergistic effects of the two. Thus, in present study, synbiotic acidophilus milk was developed satisfying functional dairy food properties. Different sets of milk were fermented with probiotic cultures (Lactobacillus acidophilus, Bifidobacterium bifidum, Lactobacillus casei, bioyoghurt culture) singly or in combination, and prebiotics namely inulin (I), oat fibre $(\mathrm{O})$ and honey $(\mathrm{H})$. Obtained 20 synbiotic samples were organoleptically tested, physico-chemically (titrable acidity percentage (TA) \& pH) and microbiologically (total viable count (TVC), coliform count and yeast \& mold count) analyzed. The incorporation of honey and inulin led to development of sweetened and low calorie sweetened synbiotic acidophilus milk, respectively. Incorporation of $B$. bifidum increased the flavour of synbiotic acidophilus milk when compared to $L$. acidophilus as control, where as $L$. casei culture showed thinner consistency in the product. Addition of prebiotic affected only the sensory scores, whereas the probiotics addition resulted in a marginal variation of pH and TA. TVC of all synbiotic acidophilus milk samples obtained were more than desirable limits for harvesting probiotic effects $\left(>10^{\wedge} 10 \mathrm{cfu} / \mathrm{ml}\right)$. Finally, a two layer feed-forward artificial neural network (ANN) was established to predict the sensory evaluation based on inputs of probiotic and prebiotic.
\end{abstract}

Keywords: Synbiotic Acidophilus Milk; Artificial Neural Network; Sensory Evaluation

\section{INTRODUCTION}

Notable fermented milks are buttermilk, acidophilus milk, bulgarian buttermilk, kumiss, kefir and yoghurt.
Acidophilus milk is a sour product that has been allowed to ferment under conditions that favor the growth and development of thermophilic lactic acid bacteria. This type of fermented milk is produced by development in milk of Lacatobacillus acidophilus. It is claimed that acidophilus milk has therapeutic and health-promoting properties. It is also claimed that the growth of Lacatobacillus acidophilus under the condition existing in the intestinal tract can replace undesirable putrefactive ermentation with a beneficial lactic fermentation [1].

Probiotics are technically defined as live microbial food ingredients that have a beneficial effect on human health. Some of the important beneficial effects are antimicrobial activity, immune system modulation, antimutagenic activity, colonization resistance activity, maintenance of micro-ecology of bowel, stimulation of Bifidobacteria, deactivation of carcinogens etc. Commercially available probiotic strains belong to genera Lactobacilli, Bifidobacterium, Streptococcus, Bacillus, Bacteriodes, Pediococcus, Leuconostoc, Propionibacteruim [2-4], Saccharomyces cerevisia and Aspergillus oryzae [5].

The other key component of functional dairy or other products is prebiotic ingredient. Prebiotics are nondigestible food ingredients that beneficially affect the host by selectively stimulating the growth and/or activity of one or a limited number of bacteria in colon that can improve the host health [6].

When prebiotics are used in combination with probiotics or live bacteria, the resultant has synergistic effects, referred to as "synbiotic". This is because in addition to the action of probiotics that promote the growth of existing strains of beneficial bacteria in the colon, prebiotics such as inulin and oligofructose also act to improve the survival, implantation and growth of newly added probiotics strains.

Inulin is a mixture of polymers consisting mainly of fructose unit; its partial enzymatic hydrolysis yields oligofructose. These are classified as prebiotic (dietary) 
fibres, which help in stabilization of foams, assist in digestion, increase stool volume, stimulate Bifidobacteria and are used for formulation of low fat preparations as fat and sugar replacers, respectively [7-9].

As a prebiotic, honey contains carbohydrates called oligosaccharides, which may improve gastrointestinal health by stimulating the growth of good bacteria in the colon. Honey has been shown to enhance growth, activity of Bifidobacteria in fermented dairy food [10, 11]. Current market trends reveal that acidophilus milk could be a suitable vehicle to develop functional dairy foods. By incorporation of variations in terms of flavour, it could be easily used in wide range of nutritional and organoleptic qualities being demanded by consumers of all age, health status, and cultures.

In the food industry, end-products must achieve a compromise between several properties, including sensory, sanitary and technological properties. Among the latter, sensory property is essential because it influences consumer choice and performance. To produce a new product, if formulation of product is achieved automatically, production speed and efficiency can be improved in addition to the increased evaluation accuracy, with an accompanying reduction in production costs $[12,13]$. Artificial neural network (ANN) could be applied to evaluate its capability in predicting product composition involved in food formulation [14-19].

Scanty information is available on incorporation of prebiotic along with probiotic in acidophilus milk. Incorporation of prebiotic and probiotic for development of functional synbiotic acidophilus milk seem to be an effective area of research. In view of the above, present investigation was undertaken to develop quality of synbiotic acidophilus milk from toned cow's milk, by use of ANN technique.

\section{MATERIALS AND METHODS}

\subsection{Materials and Chemicals}

The freeze-dried cultures of Lactobacillus acidophilus NCDC No 015, Bifidobacterium bifidum NCDC No 255 and Lactobacillus casei NCDC No 063 were obtained from Culture Collection Centre, National Dairy Research Institute, Karnal (Haryana), India. Bioyoghurt culture $(L$. bolgaricus and $S$. thermophilus) was obtained from K.C. Das Pvt. Ltd. (Bangalore). Inulin was obtained from Nutraingredients, Netherlands. Pasteurized toned cow's milk (3\% fat and 8.5\% Solid Non Fat), dietary fibre (oat), honey (Dabur India Ltd.,), skim milk powder (Amul), polyethylene cups and aluminum foil were purchased from the local market. The chemicals and media used in the present investigation were of Analytical Reagent grade.

\subsection{Activation of Cultures}

The freeze-dried cultures were activated according to the recommendations of suppliers and grown in sterile skim milk at $37 \mp 1{ }^{\circ} \mathrm{C}$ and then maintained by weekly transfers and stored at $4 \pm 1^{\circ} \mathrm{C}$ between transfers. These cultures were activated by sub culturing 3-4 times before use.

\subsection{Preparation of Synbiotic Acidophilus Milk}

Optimized level of skim milk powder (3\% wt./v) based on sensory evaluation was added to pasteurized toned milk and reheated to at $40-45^{\circ} \mathrm{C}$ Prebiotics were added at optimized level based on sensory evaluation namely inulin $(10 \% \mathrm{wt} . / \mathrm{v})$, oat fibre $(0.2 \% \mathrm{wt} . / \mathrm{v})$ and honey $(7 \%$ wt./v). The milk was inoculated with starter culture $(L$. acidophilus) as a control, and other probiotics namely $L$. casei, B. bifidum, L. bulgaricus, S. thermophilus singly or in combination with same ratio $(1: 1$ and $1: 1: 1)$ at 7 percent v./v., and then incubated at $40^{\circ} \mathrm{C}$ for $4-4 \frac{1}{2}$ hours. Different type of synbiotic acidophilus milk thus prepared were cooled to $5^{\circ} \mathrm{C}$ and stored for about 2 hours for inducing cold gelation, then stirred for better homogenous consistency. Thus, obtained symbiotic acidophilus milk samples were tested for $\mathrm{pH}$, TA, microbial analysis and sensory evaluation.

\subsection{Measurement of TA and pH of Samples}

$\mathrm{pH}$ of the synbiotic acidophilus milk was measured directly using the digital $\mathrm{pH}$ meter (Digisun Electronics, Hyderabad). TA (as \% lactic acid) of the samples was determined according to Ranganna [20].

\subsection{Total Viable Count}

$1 \mathrm{ml}$ of sample was diluted in $9 \mathrm{ml}$ distilled water and then serial dilutions were prepared. Different dilutions $\left(10^{-6}, 10^{-7}\right.$ and $\left.10^{-8}\right)$ were used to check total viable count present per $\mathrm{ml}$ on DE Man, Rogosa, Sharpe and All purpose medium with tween so agar (Hi-Media Laboratories Pvt. Ltd., Mumbai). After incubation the average count of colonies present on Petri plates were multiplied by dilution factor and expressed as colony forming units (cfu) per ml.

\subsection{Yeast \& Mold and Coliform Count}

Potato dextrose agar and Acetamide media (Hi-Media Laboratories Pvt. Ltd., Mumbai) were used to enumerate the count of yeast \& mold and coliform count, respectively. After incubation at $22 \pm 1^{\circ} \mathrm{C}$ for about a week and $37^{\circ} \mathrm{C}$ for $24-48 \mathrm{hrs}$, respectively, the average count of colonies present on petri plates, if any, were multiplied by dilution factor and expressed as colony forming units (cfu) per ml. 


\subsection{Sensory Evaluation}

Synbiotic acidophilus milk samples were evaluated for their sensory characteristics namely colour, flavour, body texture and overall acceptability (OA) using semi-trained sensory panel consisting of 10 judges drawn from faculty members of Brindavan college Banglor. The judges were requested to record their degree of liking/disliking on a scorer and using hedonic scale ranging from 1 to 9 , where 1 represented dislike extremely and 9 represented like extremely [20]. The samples were served to panelist randomly.

\subsection{Hybrid Analytical Approach}

An artificial neural network is usually defined as a network composed of a large number of processors (neurons) that are massively interconnected, operate in parallel, and learn from experience (examples). The ANN used in this research is a feed forward network that can be used to estimate a vector $\mathrm{X}$ from a measured vector $\mathrm{V}$. The ANN "learns" by adjusting the interconnection weights between layers. The answers produced by the network are repeatedly compared with the correct answers, and each time the connecting weights are adjusted slightly in the direction of the correct answers. Eventually, if the problem is learned, a stable set of weights adaptively evolves which will provide good answers for all of the sample predictions. When a new vector $\mathrm{V}$ is entered into the network, it is subtracted from the stored vectors representing cluster centers. The squares of the differences are summed and fed into a non-linear activation function to recognize the pattern which is most similar to the entered one [21].

Neural network (NN) approach is an effective method for generating new combination of input vector $P P$. Because of inherent parallel structure, a multi layer ANN can be trained for generating new combinations of prebiotics and probiotics in a very short running time. The Training set is $\left[(P P, U G), \quad P \mathrm{P} \in R^{n}, U G \in R^{4}\right]$, where $P P$ is a sample random vector for the prebiotics and probiotics based on fractional factorial experiment. Generating new combinations by neural networks helps to approximate $U G$ according to a given $P P$.

With a sigmoid activation function, a multi layer $\mathrm{NN}$ $E[U G \mid P P]$ is trained and new interpolation combination can be obtained.

The Model $O A \cong f(P P)+\varepsilon$ is a nonlinear equation that has been approximated by quadratic equation as given in Formula (1).

$$
O A=\beta_{0}+\sum_{i=1}^{P} \beta_{i} P P_{i}+\sum_{i=1}^{P} \sum_{r=i}^{P} \beta_{i r} P P_{i} P P_{r}+\varepsilon
$$

where $\beta_{0}$ is equal to the mean of $O A$ and $\beta_{i}$ and $\beta_{\text {ir }}$ are called linear and quadratic coefficients, respect tively. Moreover, the expected value of $\varepsilon$ and $O A$ to $P P$ can be recognized by Eqs. 2 and $\mathbf{3}$ respectively:

$$
\begin{gathered}
E(\varepsilon \mid \overrightarrow{P P})=0 \\
E[O A A \mid \overrightarrow{P P}]=f(\overrightarrow{P P})
\end{gathered}
$$

For obtaining the parameters of Formula (1), we minimize squared error by the following equations.

$$
\begin{aligned}
& \varepsilon_{j}=O A_{j}-\left[\beta_{0}+\sum_{i=1}^{P} \beta_{i} P P_{i j}+\sum_{i=1}^{P} \sum_{r=i}^{P} \beta_{i r} P P_{i j} P P_{r j}\right] \\
& S(\beta)=\sum_{j=1}^{n} \varepsilon_{j}^{2}= \\
& \sum_{j=1}^{n}\left(O A_{j}-\left[\beta_{0}+\sum_{i=1}^{P} \beta_{i} P P_{i j}+\sum_{i=1}^{P} \sum_{r=i}^{P} \beta_{i r} P P_{i j} P P_{r j}\right]\right)^{2}
\end{aligned}
$$

By using $\frac{\partial S(\beta)}{\partial \beta}=0$, the corresponding parameters can be determined.

For higher polynomial degree of Eq.1, we used hypothesis test as follows:

$$
\left\{\begin{array}{l}
H_{0}: \vec{\beta}=0 \\
H_{1}: \vec{\beta} \neq 0
\end{array}\right.
$$

The t-test has been used to test the degree of nonlinear equation model. For validity of approved model, we apply $F$-test for the result of actual and approximated $T D$ as Formula (7) by $F$ statistic in Formula (8).

$$
\begin{gathered}
\left\{\begin{array}{l}
H_{0}: F_{0}=F \\
H_{1}: F_{0} \neq F
\end{array}\right. \\
F=\frac{S S_{R} / P_{p}}{S S_{E} /\left(n-P_{p}-1\right)}
\end{gathered}
$$

where $S S_{R}$ is sum of squared regression results and $S S_{E}$ is the sum of squared error while $\frac{S S_{R}}{\sigma^{2}} \approx \chi_{P_{p}}^{2}$ and $\frac{S S_{E}}{\sigma^{2}} \approx \chi_{\left(n-P_{p}-1\right)}^{2}$. Hence the F statistic has a Fisher distribution with safety interval of $\alpha$, and $P_{p}$ and $\left(n-P_{p}-1\right)$ degree of freedom $\left(F_{\alpha, P_{p},\left(n-P_{p}-1\right)}\right)$.

If $F_{0} \geq F_{\alpha, P_{p},\left(n-P_{p}-1\right)}$, then $H_{0}$ is rejected and the model would be valid, otherwise the model should be modified.

\section{RESULTS AND DISCUSSIONS}

\subsection{Changes in TA and $\mathbf{p H}$}

The $\mathrm{pH}$ and TA for samples is shown in Table 1 . They remained unaffected by the type of additive used but the type of culture combination altered them. Maximum acidity and lowest $\mathrm{pH}$ were observed in case of $\mathrm{C}_{1}$ culture (Lactobacillus acidophilus), followed by $\mathrm{C}_{2}$ cultures (Lactobacillus acidophilus + Bifidobacterium bifidum), $\mathrm{C}_{3}$ cultures (Lactobacillus acidophilus + Lactobacillus 
Table 1. pH, TA and sensory score for colour, flavour, texture and OA of samples.

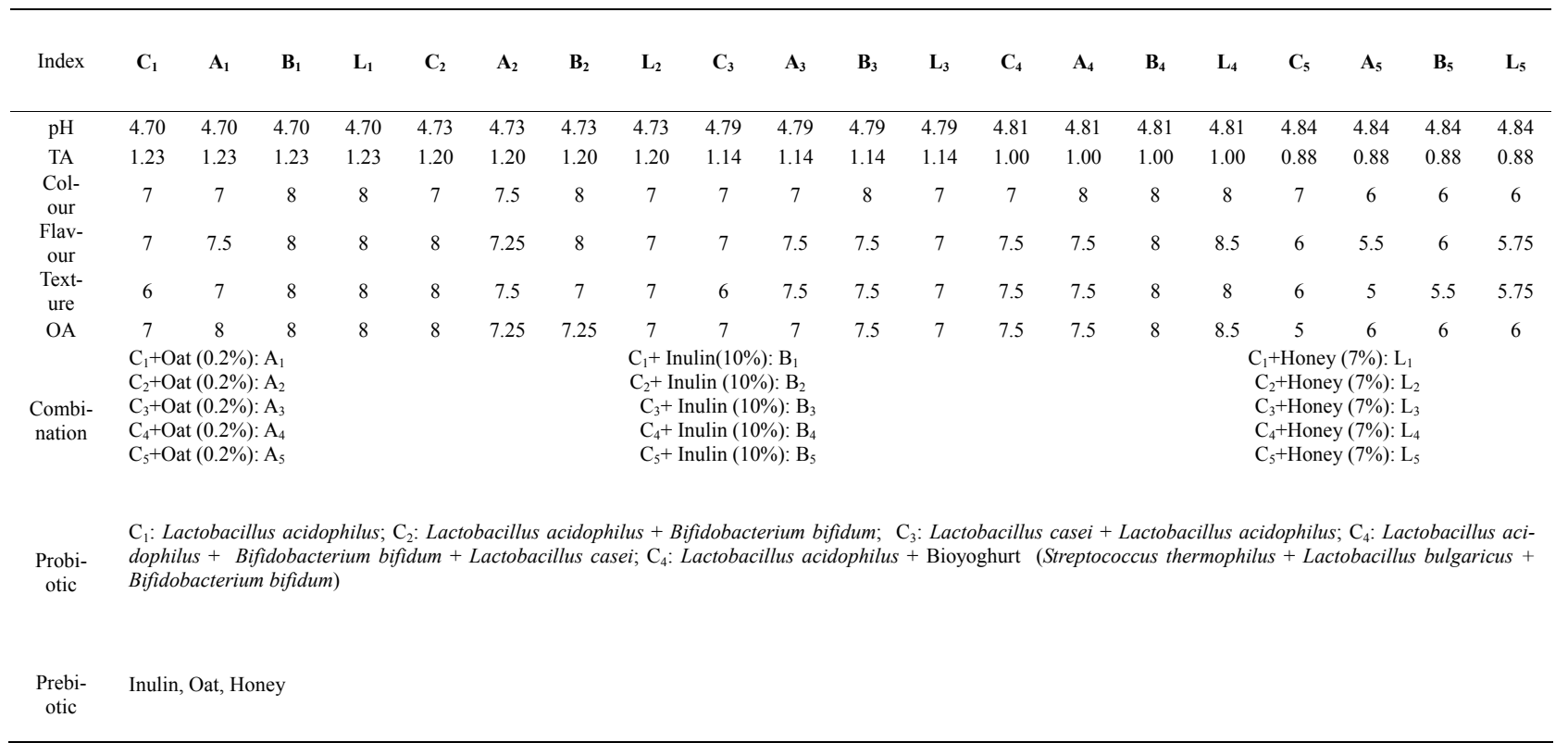

casei) cultures, $\mathrm{C}_{4}$ cultures (Lactobacillus acidophilus + Bifidobacterium bifidum + Lactobacillus casei) and $\mathrm{C}_{5}$ cultures (bioyoghurt culture). The range of $\mathrm{pH}$ and titratable acidity in synbiotic acidophilus milk was 4.70 4.84 and 1.23-0.88, respectively. Lowest TA and highest $\mathrm{pH}$ was obtained when $\mathrm{C}_{5}$ culture was used in preparation of synbiotic acidophilus milk, since it did not show acceptable quality. Similar observations were reported for different type of fermented milk by [20-24].

\subsection{Changes in TVC, Coliform and Yeast \& Mold Count}

TVC of all synbiotic acidophilus milk samples obtained ranging from $10^{8}$ to $10^{10} \mathrm{cfu} / \mathrm{ml}$, were higher than prescribed range of $10^{6}$ to $10^{8} \mathrm{cfu} / \mathrm{ml}$ at the end of storage period [25]. It can be inferred that the TVC obtained in synbiotic acidophilus milk samples in present investigation was equal to or higher than recommended range which were reported by $[26,27]$. No yeast\& mold and coliform count appeared inferring that the product developed were of good quality.

\subsection{Changes in Sensory Score}

The sensory score for colour, flavour, texture and OA for samples is shown in Table 1 . Synbiotic acidophilus milk prepared using starter culture (Lactobacillus acidophilus, B. bifidum and L. casei) and prebiotic additives (oat, inulin, honey) singly or in combination, significantly increased the colour, flavour, texture and OA of the synbiotic acidophilus milk. Addition of inulin (10\%) or honey $(7 \%)$ to $\mathrm{C}_{4}$ increased the sensory score for colour, flavour, texture and OA of the product developed. From the experimental data, it was found that $\mathrm{C}_{4}$ culture increased the sensory characteristic of the synbiotic acidophilus milk along with the honey or inulin; however addition of oat fibre did not affect the score of acidophilus milk sample much, and was not found to be as effective as inulin or honey. Thus, addition of inulin led to development of low calorie sweet acidophilus milk which is of value for recommendation to both diabetic and calorie conscious consumers.

A fractional factorial experiment combination of each element by experiment is obtained for the problem as actual UG. The training data (actual $U G$ ) are put into a neural network and the output of ANN is obtained as UG (NN). The structure of proposed ANN is as follows: An 8 element vector is considered as input layer of the network which are the most effective factors in the product specifications. And the output layer is included a 4 element vector. For instance, the input vector $[1,0,1,0,0$, $0,0.002,0]$ indicates that one unit of L.c and one unite of L.a combined with 0.002 unit oat cause the output vector of $[7,7.5,7.5,7]$ which represents a product with the score of 7, 7.5, 7.5, 7 for colour, flavour, texture and OA, respectively, in laboratory samples. Whilst the output of the proposed ANN is [7.3408, 7.3928, 7.1035, 7.3896]. The deviation between the output of $\mathrm{NN}$ and the observed actual $U G(\Delta)$ is reported in Table 2. Moreover the treatment of $\Delta$ is shown in Figure 1. Two layer ANN has been developed to generate fractional factorial experiment of $(P P, U G)$

Two layer feed forward NN has been trained to generate new fractional factorial experiment of $(P P, U G)$. The 
Table 2. ANN Error $(\Delta)$, Actual and NN Output for different combinations.

\begin{tabular}{cccc}
\hline Input & Actual Output & NN Output & NN Error \\
\hline$[1 ; 0 ; 0 ; 0 ; 0 ; 0 ; 0 ; 0]$ & {$[7 ; 7 ; 6 ; 7]$} & {$[7.2743 ; 7.422 ; 7.0387 ; 7.4512]$} & {$[-0.27433 ;-0.422 ;-1.0387 ;-0.4512]$} \\
{$[1 ; 1 ; 0 ; 0 ; 0 ; 0 ; 0 ; 0]$} & {$[7 ; 8 ; 8 ; 8]$} & {$[7.4576 ; 7.7008 ; 7.4737 ; 7.6375]$} & {$[-0.45762 ; 0.29923 ; 0.52627 ; 0.36247]$} \\
{$[1 ; 0 ; 1 ; 0 ; 0 ; 0 ; 0 ; 0]$} & {$[7 ; 7 ; 6 ; 7]$} & {$[7.3409 ; 7.3932 ; 7.1037 ; 7.3898]$} & {$[-0.34092 ;-0.39315 ;-1.1037 ;-0.38982]$} \\
{$[1 ; 1 ; 1 ; 0 ; 0 ; 0 ; 0 ; 0]$} & {$[7 ; 7.5 ; 7.5 ; 7.5]$} & {$[7.5242 ; 7.6719 ; 7.5386 ; 7.5762]$} & {$[-0.5242 ;-0.17192 ;-0.038638 ;-0.07615]$} \\
{$[1 ; 1 ; 0 ; 1 ; 1 ; 0 ; 0 ; 0]$} & {$[7 ; 7.5 ; 7 ; 7.25]$} & {$[6.2442 ; 6.298 ; 5.7955 ; 6.4128]$} & {$[0.75584 ; 1.202 ; 1.2045 ; 0.83723]$} \\
{$[1 ; 0 ; 0 ; 0 ; 0 ; 0 ; 0.002 ; 0]$} & {$[7 ; 7.5 ; 7 ; 8]$} & {$[7.2742 ; 7.4217 ; 7.0386 ; 7.451]$} & {$[-0.27421 ; 0.078317 ;-0.038606 ; 0.54898]$} \\
{$[1 ; 1 ; 0 ; 0 ; 0 ; 0 ; 0.002 ; 0]$} & {$[7.5 ; 7.25 ; 7.5 ; 7.25]$} & {$[7.4575 ; 7.7005 ; 7.4736 ; 7.6373]$} & {$[0.042505 ;-0.45045 ; 0.026408 ;-0.38735]$} \\
{$[1 ; 0 ; 1 ; 0 ; 0 ; 0 ; 0.002 ; 0]$} & {$[7 ; 7.5 ; 7.5 ; 7]$} & {$[7.3408 ; 7.3928 ; 7.1035 ; 7.3896]$} & {$[-0.34079 ; 0.10716 ; 0.39649 ;-0.38964]$} \\
{$[1 ; 1 ; 1 ; 0 ; 0 ; 0 ; 0.002 ; 0]$} & {$[8 ; 7.5 ; 7.5 ; 7.5]$} & {$[7.5241 ; 7.6716 ; 7.5385 ; 7.576]$} & {$[0.47592 ;-0.1716 ;-0.0385 ;-0.075966]$} \\
{$[1 ; 1 ; 0 ; 1 ; 1 ; 0 ; 0.002 ; 0]$} & {$[6 ; 5.5 ; 5 ; 6]$} & {$[6.244 ; 6.2977 ; 5.7953 ; 6.4126]$} & {$[-0.24404 ;-0.79767 ;-0.79534 ;-0.41259]$} \\
{$[1 ; 0 ; 0 ; 0 ; 0 ; 0.1 ; 0 ; 0]$} & {$[8 ; 8 ; 8 ; 8]$} & {$[7.5089 ; 7.5088 ; 7.1325 ; 7.4538]$} & {$[0.49114 ; 0.49117 ; 0.86746 ; 0.54621]$} \\
{$[1,1,0,0,0,0.1,0,0]$} & {$[8,8,7,7.25]$} & {$[7.6921 ; 7.7876 ; 7.5675 ; 7.6401]$} & {$[0.30786 ; 0.2124 ;-0.56753 ;-0.39012]$} \\
{$[1,0,1,0,0,0.1,0,0]$} & {$[8,7,7.5,7.5]$} & {$[7.5754 ; 7.48 ; 7.1975 ; 7.3924]$} & {$[0.42456 ; 0.020018 ; 0.30255 ; 0.10759]$} \\
{$[1,1,1,0,0,0.1,0,0]$} & {$[8,8,8,8]$} & {$[7.7587 ; 7.7587 ; 7.6324 ; 7.5787]$} & {$[0.24127 ; 0.24125 ; 0.36756 ; 0.42126]$} \\
{$[1,1,0,1,1,0.1,0,0]$} & {$[6,6,5.5,6]$} & {$[6.4787 ; 6.3848 ; 5.8893 ; 6.4154]$} & {$[-0.47868 ;-0.38481 ;-0.38928 ;-0.41536]$} \\
{$[1,0,0,0,0,0,0,0.07]$} & {$[8,8,8,8]$} & {$[7.3097 ; 7.4554 ; 7.1605 ; 7.5169]$} & {$[0.69029 ; 0.54465 ; 0.83949 ; 0.48314]$} \\
{$[1,1,0,0,0,0,0,0.07]$} & {$[7,7,7,7]$} & {$[7.493 ; 7.7341 ; 7.5955 ; 7.7032]$} & {$[-0.493 ;-0.73412 ;-0.5955 ;-0.70319]$} \\
{$[1,0,1,0,0,0,0,0.07]$} & {$[7,7,7,7]$} & {$[7.3763 ; 7.4265 ; 7.2254 ; 7.4555]$} & {$[-0.3763 ;-0.42651 ;-0.22542 ;-0.45548]$} \\
{$[1,1,1,0,0,0,0,0.07]$} & {$[8,8.5,8,8.5]$} & {$[7.5596 ; 7.7053 ; 7.6604 ; 7.6418]$} & {$[0.44041 ; 0.79473 ; 0.33959 ; 0.85819]$} \\
{$[1,1,0,1,0,1,0,0.07]$} & {$[6,5.75,5.75,6]$} & {$[6.0662 ; 5.789 ; 5.7895 ; 6.0184]$} & {$[-0.066229 ;-0.039021 ;-0.039541 ;-0.018398]$} \\
\hline
\end{tabular}

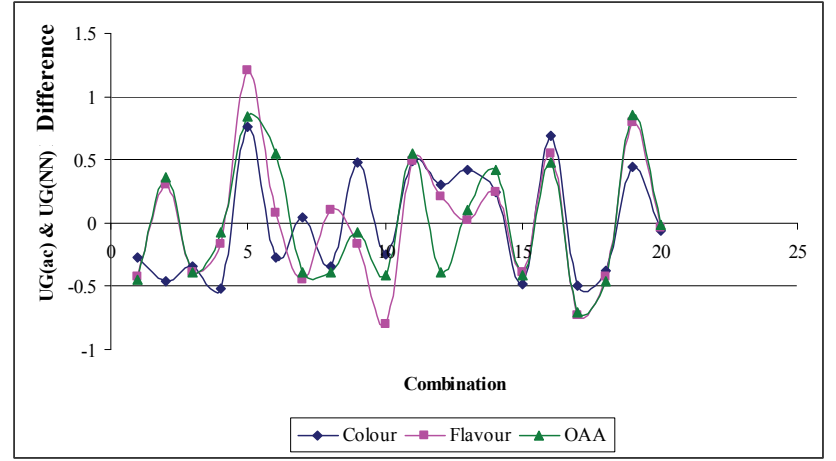

Figure 1. $\Delta$ Treatment.

block diagram of proposed NN has been illustrated in Figure 3. Two non-linear activation functions were dedicated to each layer as Logarithmic and Perlin for first and second layers, respectively. Table 3 shows the simulated $O A$ obtained by ANN for different combinations of probiotic and prebiotic. Block diagram of proposed ANN has been illustrated in Figure 2.

By using proposed approach, the equation $O A=$ $f(L . a, B . b, L . c, L . b, S . t, I, O, H)$ is approximated as follows:

$$
\begin{aligned}
O A= & 7.829(\text { L.a })-0.629(\text { L.b })-0.372(S . t) \\
- & 4428.58(O)^{2}+184.60(\text { B.b })(O) \\
& +4.80(\text { L.c })(I)-99.72(\text { L.b })(O)-5.14(S . t)(H)
\end{aligned}
$$

Table 4 indicates the t-test analysis results for the coefficients of the above-mentioned quadratic equation.

With the equation which approximates OA in terms of probiotic and prebiotic, as it is observed from the results reported in Table 4 , the p-values of $B . b, \mathrm{I}, \mathrm{O}, \mathrm{H}, \mathrm{I}^{2}$, $\mathrm{H}^{2}$, and some of other components are greater than 0.360 . This means that with a confidence of $\% 64$, these factors are not important in approximating OA and can be safely removed from further consideration.

For optimization, the following quadratic mathematical model has been solved by LINGO 8 software package.

$$
\begin{gathered}
\text { Max OA }=7.829(L . a)-0.629(L . b)-0.372(S . t) \\
-4428.58(O)^{2}+184.60(B . b)(O)+4.80(\text { L.c })(I) \\
-99.72(L . b)(O)-5.14(S . t)(H) \\
0 \leq I \leq 0.12 \\
0 \leq O \leq 0.004 \\
0 \leq H \leq 0.1 \\
L a, B b, L b, S t, L c \in\{0,1\}
\end{gathered}
$$

which lead to $[1,1,1,0,0,0.09,0.002,0.075]$ as the optimum combination of prebiotic and probiotic.

\section{CONCLUSIONS}

From the results of the experiments it can be concluded that the addition of inulin or honey had synergistic effect 
on the physico-chemical and sensory quality of probiotic acidophilus milk. Also a satisfactorily good quality synbiotic acidophilus milk could be prepared by fermenting milk with combinations of all cultures $\left(\mathrm{C}_{4}\right.$, Lactobacillus acidophilus, Bifidobacterium bifidum and Lactobacillus casei) along with 7 percent honey (sweetened acidophilus milk) or 10 percent inulin (for low calorie sweetened acidophilus milk). Moreover, in this research an ar- tificial neural network has been applied to create more experimental data. Then by developing a quadratic mathematical model, the optimum value of OA has been approximated with respect to probiotics and prebiotics, i.e. combination of Lactobacillus acidophilus, Bifidobacterium bifidum and Lactobacillus casei along with 7.5 percent honey, 9 percent inulin and 0.2 percent oat fibre.

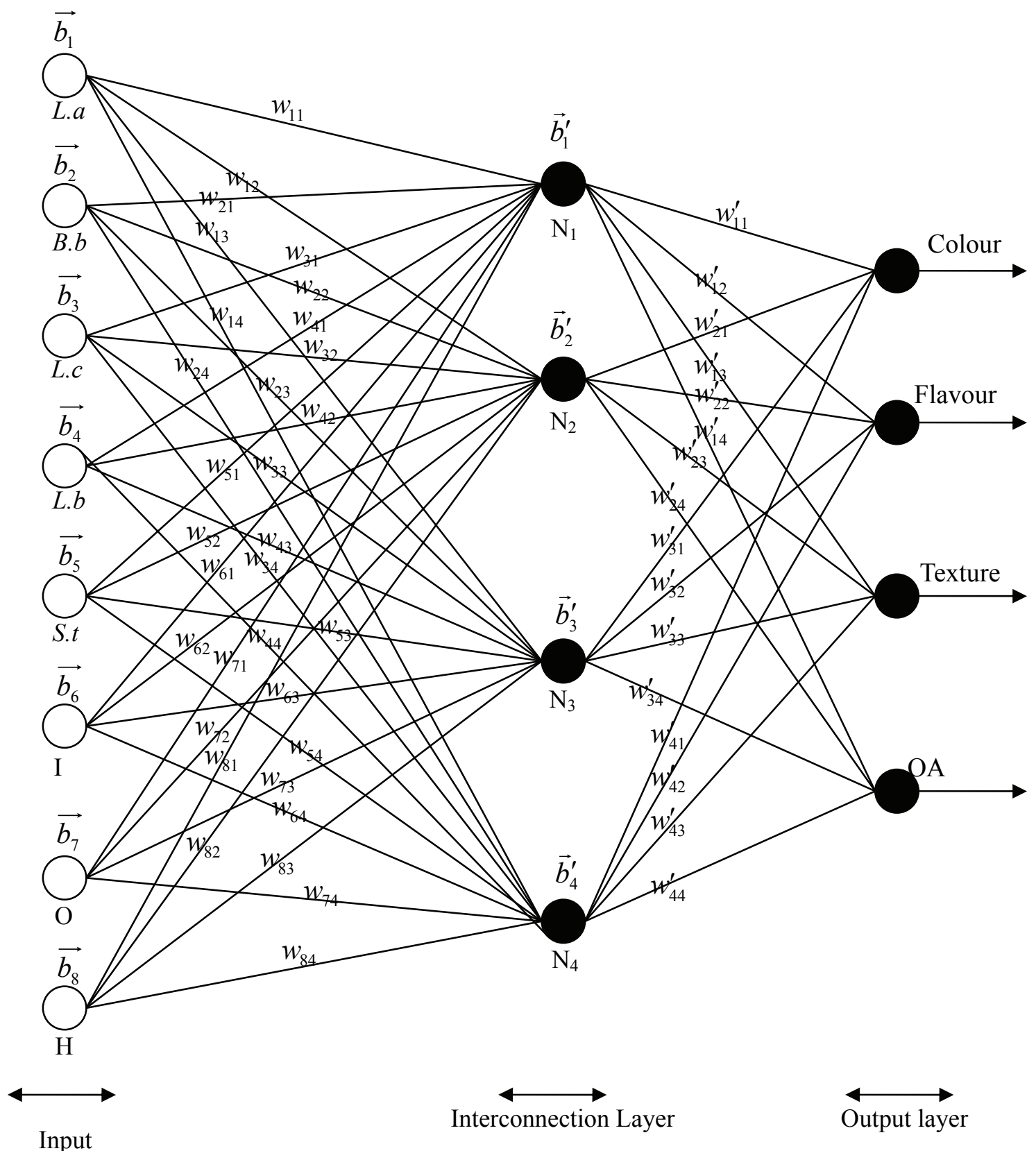

Figure 2. Block diagram of proposed NN. 
Table 3. ANN output.

\begin{tabular}{|c|c|c|c|c|c|c|c|c|c|c|c|c|c|c|c|c|c|c|}
\hline L.a & $B . b$ & L.c & $L . b$ & S.t & I & $\mathrm{O}$ & $\mathrm{H}$ & $\mathrm{OA}$ & L.a & $B . b$ & & L.c & L.b & S.t & I & $\mathrm{O}$ & $\mathrm{H}$ & $\mathrm{OA}$ \\
\hline 1 & 1 & 0 & 1 & 0 & 0.12 & 0.003 & 0.05 & 6.451636804 & 1 & 1 & & 1 & 1 & 0 & 0.08 & 0 & 0.05 & 7.484155 \\
\hline 1 & 0 & 1 & 0 & 1 & 0.12 & 0.004 & 0.07 & 6.101418984 & 1 & 1 & & 1 & 0 & 0 & 0.12 & 0.001 & 0.08 & 8.346724 \\
\hline 1 & 0 & 0 & 1 & 1 & 0.06 & 0.004 & 0.01 & 5.751022455 & 1 & 1 & & 0 & 0 & 0 & 0.02 & 0.003 & 0.02 & 7.528115 \\
\hline 1 & 0 & 1 & 0 & 0 & 0.02 & 0.001 & 0.06 & 6.984832124 & 1 & 1 & & 0 & 0 & 1 & 0.06 & 0.001 & 0.03 & 7.535261 \\
\hline 1 & 0 & 0 & 0 & 1 & 0.06 & 0.004 & 0.05 & 6.435936602 & 1 & 0 & & 0 & 1 & 0 & 0 & 0.002 & 0.09 & 6.791768 \\
\hline 1 & 0 & 1 & 1 & 0 & 0.1 & 0.003 & 0 & 6.149605379 & 1 & 1 & & 0 & 0 & 1 & 0.04 & 0.003 & 0.1 & 7.339067 \\
\hline 1 & 1 & 0 & 1 & 1 & 0.1 & 0.002 & 0.03 & 5.752972025 & 1 & 1 & & 1 & 0 & 0 & 0.12 & 0.002 & 0.09 & 8.346724 \\
\hline 1 & 0 & 0 & 1 & 0 & 0.08 & 0.001 & 0.07 & 6.854626756 & 1 & 1 & & 1 & 0 & 0 & 0.02 & 0.001 & 0.04 & 8.154417 \\
\hline 1 & 0 & 1 & 1 & 1 & 0.06 & 0.004 & 0.08 & 4.65789474 & 1 & 1 & & 0 & 1 & 0 & 0.06 & 0.002 & 0.04 & 7.423626 \\
\hline 1 & 1 & 1 & 1 & 0 & 0.04 & 0.001 & 0.05 & 7.48414076 & 1 & 1 & & 0 & 0 & 0 & 0.1 & 0.003 & 0.1 & 7.536114 \\
\hline 1 & 1 & 0 & 1 & 1 & 0.06 & 0.003 & 0.05 & 5.75163863 & 1 & 1 & & 0 & 0 & 1 & 0.1 & 0.002 & 0 & 7.532254 \\
\hline 1 & 0 & 1 & 1 & 1 & 0.14 & 0.002 & 0.09 & 6.074391253 & 1 & 0 & & 1 & 1 & 1 & 0.12 & 0.001 & 0.06 & 6.577422 \\
\hline 1 & 0 & 1 & 0 & 0 & 0.12 & 0.003 & 0.01 & 7.006773357 & 1 & 1 & & 0 & 1 & 1 & 0.08 & 0.001 & 0.05 & 5.757191 \\
\hline 1 & 0 & 1 & 0 & 0 & 0.1 & 0.001 & 0.05 & 7.362838031 & 1 & 1 & & 0 & 1 & 0 & 0.08 & & 0.04 & 7.077645 \\
\hline 1 & 0 & 1 & 1 & 0 & 0.08 & 0.001 & 0.04 & 6.633418746 & 1 & 0 & & 1 & 1 & 0 & 0.06 & & 0.06 & 6.145 \\
\hline 1 & 0 & 1 & 1 & 1 & 0.08 & 0.001 & 0.04 & 6.28 & 1 & 1 & & 1 & 1 & 0 & 0.02 & & 0.1 & 165 \\
\hline 1 & 1 & 1 & 0 & 1 & 0.12 & 0 & 0.06 & 7.53 & 1 & 1 & & 1 & 0 & 0 & 0.1 & & 0.01 & \\
\hline 1 & 0 & 0 & 0 & 1 & 0 & 0.003 & 0.1 & 6.93695 & 1 & 1 & & 1 & 1 & 1 & 0.14 & & 0.04 & 7.568186 \\
\hline 1 & 1 & 1 & 0 & 0 & 0.02 & 0.003 & 0.03 & 7.746431306 & 1 & 1 & & 0 & 1 & 0 & 0.08 & & 0.1 & 6.09158 \\
\hline 1 & 1 & 1 & 0 & 1 & 0.04 & 0.002 & 0.09 & 7.536085487 & 1 & 1 & & 1 & 0 & 1 & 0.12 & & 0.09 & 7.536147 \\
\hline 1 & 1 & 0 & 1 & 1 & 0.02 & 0.001 & 0.06 & 5.772025264 & 1 & 1 & & 1 & 0 & 1 & 0.08 & & 0.04 & 7.535996 \\
\hline 1 & 1 & 0 & 1 & 0 & 0 & 0 & 0.03 & 7.483844669 & 1 & 1 & & 0 & 1 & 0 & 0.12 & & 0.07 & 7.26907 \\
\hline 1 & 0 & 0 & 1 & 0 & 0 & 0.002 & 0.06 & 7.207824048 & 1 & 0 & & 0 & 0 & 0 & 0.08 & & 0.01 & 7.536163 \\
\hline 1 & 0 & 0 & 0 & 1 & 0 & 0.001 & 0.06 & 7.52845751 & 1 & 1 & & 1 & 1 & 1 & 0.1 & & 0.03 & 7.549508 \\
\hline 1 & 1 & 1 & 0 & 0 & 0.06 & 0.001 & 0.02 & 8.056513417 & 1 & 1 & & 1 & 0 & 0 & 0.04 & & 0.05 & 8.315503 \\
\hline 1 & 1 & 1 & 0 & 0 & 0.08 & 0.001 & 0.08 & 8.346682 & 1 & 0 & c & & & 0.12 & $2 \quad 0.003$ & 0.01 & & 124803383 \\
\hline 1 & 1 & 1 & 0 & 1 & 0.08 & 0.003 & 0.01 & 7.536134 & 1 & 0 & ( & ( & 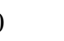 & 0.06 & 0.003 & 0.06 & & 935897712 \\
\hline 1 & 0 & 0 & 0 & 1 & 0.12 & 0.002 & 0.1 & 6.198301 & 1 & 1 & c & ( & & 0.04 & 0.003 & 0.03 & & .535469986 \\
\hline 1 & 0 & 1 & 1 & 0 & 0.08 & 0.004 & 0.06 & 5.912921 & 1 & 1 & 1 & & & 0.12 & 0.004 & 0.01 & & .504576323 \\
\hline 1 & 0 & 0 & 0 & 0 & 0.06 & 0.002 & 0.06 & 7.536133 & 1 & 1 & 1 & ( & & 0.1 & 0.003 & 0.07 & & 346595236 \\
\hline 1 & 0 & 0 & 1 & 0 & 0.08 & 0.001 & 0.06 & 7.011749 & 1 & 1 & 1 & & & 0.06 & 0.001 & 0 & & .484170893 \\
\hline 1 & 1 & 0 & 1 & 1 & 0.04 & 0.002 & 0.03 & 5.760403 & 1 & 0 & 1 & ( & & 0.12 & 0.001 & 0.09 & & 896582959 \\
\hline 1 & 0 & 0 & 0 & 1 & 0.1 & 0.001 & 0.01 & 7.521084 & 1 & 1 & 1 & ( & & 0.06 & 0.002 & 0.02 & & .536179995 \\
\hline 1 & 0 & 0 & 0 & 1 & 0.1 & 0.001 & 0.03 & 7.501948 & 1 & 1 & 0 & & & 0.14 & 0.001 & 0.01 & & .762277224 \\
\hline 1 & 1 & 1 & 1 & 0 & 0.12 & 0.002 & 0.08 & 7.47813 & 1 & 1 & 1 & ( & & 0 & 0.002 & 0.02 & & .536186589 \\
\hline 1 & 1 & 0 & 0 & 1 & 0.1 & 0.002 & 0.05 & 7.505374 & 1 & 1 & c & & & 0.08 & 0.004 & 0.09 & & .845121956 \\
\hline 1 & 0 & 0 & 0 & 0 & 0.04 & 0.003 & 0.08 & 7.534859 & 1 & 0 & 0 & ( & & 0.02 & 0.002 & 0.07 & & .534845748 \\
\hline 1 & 1 & 1 & 0 & 1 & 0.12 & 0.004 & 0.02 & 7.534946 & 1 & 0 & c & ( & & 0.08 & 0.001 & 0.1 & & .234711688 \\
\hline 1 & 1 & 1 & 1 & 1 & 0.04 & 0.002 & 0.02 & 7.363935 & 1 & 1 & c & & & 0.02 & 0 & 0.03 & & 090998447 \\
\hline 1 & 1 & 0 & 0 & 1 & 0.12 & 0.001 & 0.03 & 7.531196 & 1 & 0 & c & & & 0 & 0.003 & 0.05 & & .822632654 \\
\hline 1 & 1 & 0 & 1 & 1 & 0.12 & 0.001 & 0.05 & 5.7 & 1 & 0 & 0 & 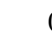 & & 0.12 & 0.001 & 0.06 & & .346438551 \\
\hline 1 & 0 & 1 & 1 & 0 & 0.06 & 0.003 & 0. & & 1 & 0 & & 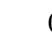 & & 0.12 & 0.002 & 0.1 & & .210684753 \\
\hline 1 & 0 & 0 & 0 & 1 & 0.02 & 0.002 & 0.01 & & 1 & 1 & 0 & & & 0.08 & 0.003 & 0.08 & & 399538417 \\
\hline 1 & 1 & 0 & 1 & 0 & 0.08 & 0.004 & 0.03 & & 1 & 0 & c & 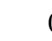 & & 0.02 & 0 & 0.08 & & .530073062 \\
\hline 1 & 1 & 0 & 0 & 0 & 0.14 & 0 & 0.03 & & 1 & 1 & & 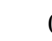 & & 0.06 & 0.002 & 0.01 & & .535503254 \\
\hline 1 & 1 & 0 & 0 & 0 & 0.1 & 0.004 & 0.08 & 7.53603 & 1 & 1 & & ( & & 0.02 & 0.004 & 0.03 & & .533041896 \\
\hline 1 & 1 & 0 & 0 & 0 & 0.04 & 0.003 & 0.1 & 7.536176 & 1 & 1 & & ( & & 0.02 & 0.002 & 0.06 & & .531179784 \\
\hline 1 & 0 & 1 & 1 & 1 & 0.04 & 0.003 & 0.03 & 5.24282 & 1 & 0 & & & & 0.06 & 0 & 0.08 & & 651366866 \\
\hline 1 & 1 & 1 & 0 & 1 & 0.08 & 0.003 & 0.02 & 7.536106 & 1 & 0 & 0 & 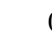 & & 0.12 & 0.003 & 0.03 & & .536097338 \\
\hline 1 & 1 & 0 & 1 & 1 & 0.1 & 0.003 & 0.09 & 5.751219 & 1 & 1 & 0 & & & 0.1 & 0.002 & 0.07 & & .751497642 \\
\hline
\end{tabular}

Table 4. The t-test and P-value analysis results for the coefficients.

\begin{tabular}{lcccccccccccccc}
\hline $\begin{array}{l}\text { Coeffi- } \\
\text { cients }\end{array}$ & $B . b$ & L.b & S.t & $\mathrm{I}$ & $\mathrm{O}$ & $\mathrm{H}$ & $\mathrm{I}^{2}$ & $\mathrm{O}^{2}$ & $\mathrm{H}^{2}$ & $\mathrm{IO}$ & $\mathrm{IH}$ & $\mathrm{OH}$ \\
T Stat & -0.298 & -2.084 & -1.247 & -0.811 & 0.158 & 0.418 & 0.781 & -1.388 & 0.223 & -0.347 & -0.813 & -0.758 \\
P-value & 0.76 & 0.04 & 0.21 & 0.42 & 0.87 & 0.67 & 0.43 & 0.16 & 0.82 & 0.72 & 0.41 & 0.45 \\
\hline $\begin{array}{l}\text { Coeffi- } \\
\text { cients }\end{array}$ & B.b.I & B.b.O & B.b.H & L.c.I & L.c.O & L.c.H & L.b.I & L.b.O & L.b.H & S.t.I & S.t.O & S.t.H \\
T Stat & 0.477 & 2.045 & 0.217 & 1.974 & -0.963 & 0.442 & -0.669 & -1.285 & -0.661 & 0.187 & 0.235 & -1.59 \\
P-value & 0.63 & 0.04 & 0.82 & 0.05 & 0.33 & 0.65 & 0.5 & 0.2 & 0.51 & 0.85 & 0.81 & 0.11 \\
\hline
\end{tabular}




\section{REFERENCES}

[1] De Roos, N.M. and Katan, M.B. (1998) Effects of probiotic bacteria on diarrhea, lipid metabolism, and carcinogenesis: A review of papers published between 1988 and 1998. American Journal of Clinical Nutrition, 71(2), 405-411.

[2] Meile, L., Blay, G.L. and Thierry, A. (2008) Safety assessment of dairy microorganisms: Propionibacterium and Bifidobacterium. International Journal of Food Microbiology, 126(3), 316-320.

[3] Tharmaraj, N., Nagendra, P. and Shah, N.P. (2004) Survival of Lactobacillus acidophilus, Lactobacillus paracasei subsp. paracasei, Lactobacillus rhamnosus, Bifidobacterium animalis and Propionibacterium in cheesebased dips and the suitability of dips as effective carriers of probiotic bacteria. International Dairy Journal, 14(12), 1055-1066.

[4] Douglas, L.C. and Sanders, M.E. (2008) Probiotics and prebiotics in dietetics practice. Journal of the American Dietetic Association, 108(3), 510-521.

[5] Verma, S.V.S. and Singh, R. (1995) Probiotic and their role in broiler chicken production: An overview. Journal of Animal Science, 10, 359-363.

[6] Niness, K.R. (1999) Inulin and oligofructose. What are they? Journal of Nutrition, 129(7), 1402-1406.

[7] Jenkins, D.J.A., Kendall, C.W.C. and Vladimir, V. (1999) Effect of inulin and oligofructose on intestinal flora. Journal of Nutrition, 5(2), 1431-1433.

[8] Villaluenga, C.M., Frías, J., Gómez, R. and Valverde, C.V. (2006) Influence of addition of raffinose family oligosaccharides on probiotic survival in fermented milk during refrigerated storage. International Dairy Journal, 16(7), 768-774.

[9] Zárate, G., Chaia, A.P. and Oliver, G. (2002) Some characteristics of practical relevance of the $\beta$-galactosidase from potential probiotic strains of propionibacterium acidipropionici, Anaerobe, 8(5), 259-267.

[10] Mehanna, N.S., Salem, M.M.E., Zaky, W.M. and ElKhalek, A.B.A. (2003) Viability of probiotic bacteria in functional fermented milk. Annals of Agricultural Science, 48, 691-702.

[11] Sanz, M.L., Polemis, N., Morales, V., Corzo, N., Dvakoularakou, A. and Gibson, G.R. (2005) In vitro investigation of potential prebiotic activity of honey oligosaccharides. Journal of Agricultural and Food Chemistry, 11(3), 2914-2921.

[12] Sun, D.W. and Brosnan, T. (2003) Pizza quality evaluation using computer vision-Part 1 Pizza base and sauce spread. Journal of Food Engineering, 57(1), 81-89.

[13] Sun, D.W. and Brosnan, T. (2003) Pizza quality evaluation using computer vision-Part 2 Pizza topping analysis. Journal of Food Engineering, 57(1), 91-95.

[14] Gnanasekharan, V. and Floros, J.D. (1995) Back propagation neural networks: Theory and applications for food science and technology. Developments in Food Science, 37(2), 2151-2168.

[15] Eerikäinen, T., Linko, P., Linko, S., Siimes, T. and Zhu, Y.H. (1993) Fuzzy logic and neural network applications in food science and technology. Trends in Food Science \& Technology, 4(8), 237-242.

[16] Hernández, J.A. (2008) Optimum operating conditions for heat and mass transfer in foodstuffs drying by means of neural network inverse. Food Control, 20(4), 435-438.

[17] Zenoozian, M.S. and Devahastin, S. (2009) Application of wavelet transform coupled with artificial neural network for predicting physicochemical properties of osmotically dehydrated pumpkin. Journal of Food Engineering, 90(2), 219-227.

[18] Zeng, X., Ruan, D. and Koehl, L. (2008) Intelligent sensory evaluation: Concepts, implementations, and applications. Mathematics and Computers in Simulation, 77 (5-6), 443-452.

[19] Munkevik, P., Hall, G. and Duckett, T. (2007) A computer vision system for appearance-based descriptive sensory evaluation of meals. Journal of Food Engineering, 78(1), 246-256.

[20] Ranganna, S. (2002) Handbook of analysis and quality control for fruit and vegetable products. Tata McGrawHill, New Delhi.

[21] Chen, M.J, Chen, K.N. and Kin, C.W. (2006) Development and verification of an optimum composition model for a symbiotic fermented milk using sequential quadratic programming techniques, Asian-Australasian Journal of Animal Sciences, 19(10), 1490-1495.

[22] Amiri, Z.R. (2001) Optimization of parameters for low fat prebiotic quality yoghurt using RSM and to study the effect of probiotic cultures during its storage. Thesis, Doctor of Philosophy, G.B. Pant University of Agriculture and Technology, Pantnagar.

[23] Khandelwal, P. (2002) Studies on the preparation of symbiotic yoghurt-cheese from buffalo milk. Thesis, Doctor of Philosophy, G.B. Pant University of Agriculture and Technology, Pantnagar.

[24] Oliveira, M.N., Sodini, I., Remeuf, F. and Corrieu, G. (2001) Effect of milk supplementation and culture composition on acidification, textural properties and microbiological stability of fermented milks containing probiotic bacteria. International Dairy Journal, 11(11-12), 935-942.

[25] Salminen, S., Bouley, C. and Boutron-Ruault, M.C. (1998) Gastrointestinal physiology and function-Targets for functional food development. British Journal of Nutrition, 80(Suppl 1), S147-S171.

[26] Kim, H.S. (1998) Characterization of lactabacilli and bifidobacteria as applied to dietary adjuncts. Journal of Dairy Science, 23, 6-9.

[27] Vinderola, C.G., Costa, G.A., Regenhardt, S. and Reinheimer, J.A. (2002) Influence of compounds associated with fermented dairy products on the growth of lactic acid starter and probiotic bacteria. International Dairy Journal, 12(7), 579-589. 